\title{
From first symptoms to final diagnosis of Cushing's disease: experiences of 176 patients
}

\author{
llonka Kreitschmann-Andermahr ${ }^{1}$, Tsambika Psaras ${ }^{2}$, Maria Tsiogka ${ }^{3}$, Daniel Starz ${ }^{4}$, \\ Bernadette Kleist ${ }^{1}$, Sonja Siegel ${ }^{1,4}$, Monika Milian ${ }^{5}$, Johannes Kohlmann ${ }^{4}$, \\ Christa Menzel ${ }^{4}$, Dagmar Führer-Sakel ${ }^{3}$, Jürgen Honegger $^{5}$, Ulrich Sure ${ }^{1}$, \\ Oliver Müller ${ }^{1}$ and Michael Buchfelder ${ }^{4}$
}

${ }^{1}$ Department of Neurosurgery, University Hospital Essen, University of Essen-Duisburg, Hufelandstrasse 55, 45147 Essen, Germany, ${ }^{2}$ Department of Neurosurgery, Hospital of Cologne-Merheim, Cologne, Germany, ${ }^{3}$ Department of Endocrinology, University of Essen-Duisburg, Essen, Germany, ${ }^{4}$ Department of Neurosurgery, University of ErlangenNuremberg, Erlangen, Germany and ${ }^{5}$ Department of Neurosurgery, University of Tuebingen, Tuebingen, Germany
Correspondence should be addressed to I Kreitschmann-Andermahr Email

ilonka.kreitschmann@ uk-essen.de

The authors and the journal apologise for an error in Figure 1 in the above article published in the European Journal of Endocrinology vol 172, issue 3, pp 285-289. In the figure, the reasons for medical consultation of an internist before the diagnosis of Cushing's disease have been erroneously presented to be the same as to those for consultation of a gynaecologist. The figure legend had also been inadvertently cropped. The correct figure with the legend is published in full below.

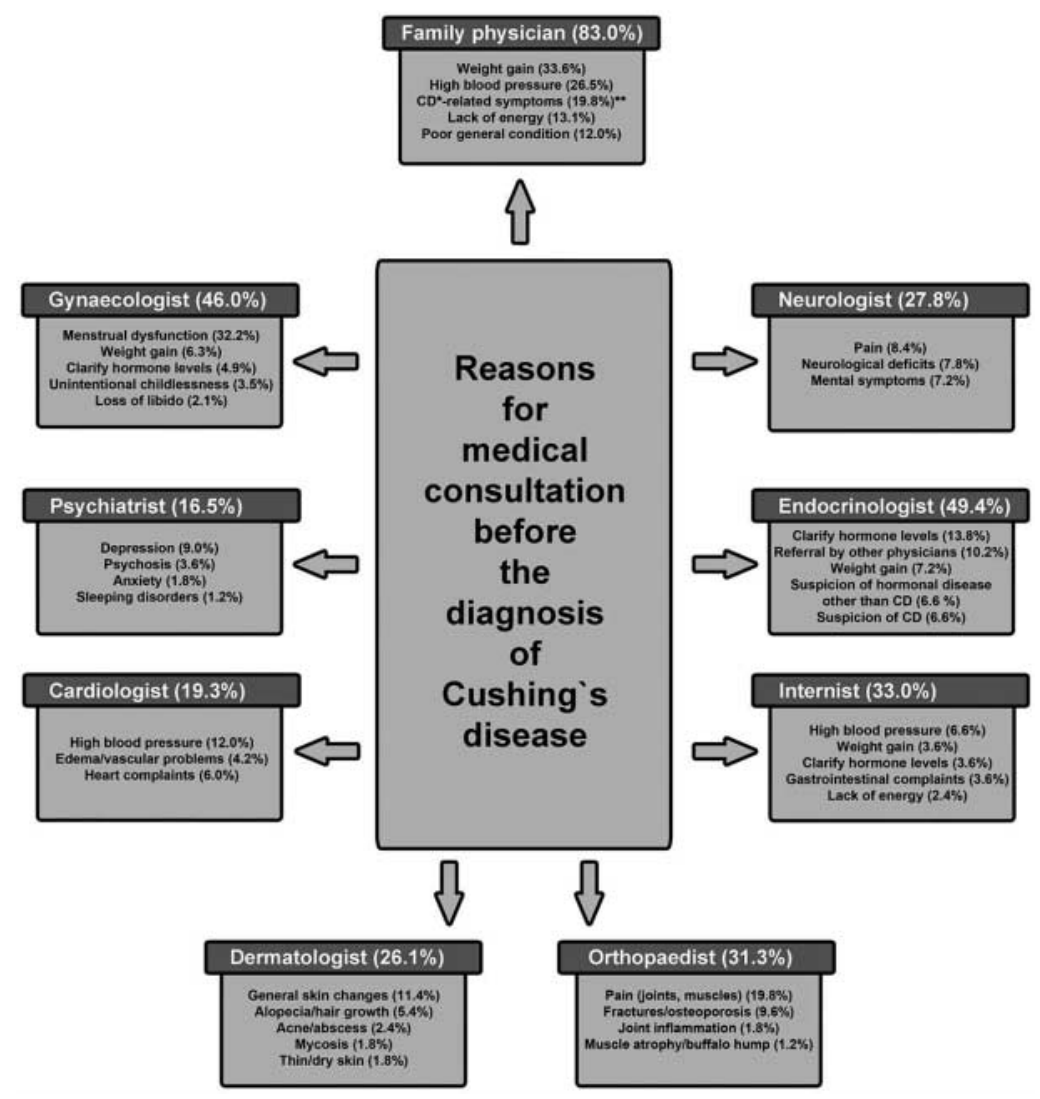

\section{Figure 1}

Most frequent symptom categories and the medical specialists to whom they were presented. *CD, Cushing's disease. **This (c) 2015 European Society of Endocrinology Printed in Great Britain cluster contains symptoms such as buffalo-hump, moon-face, plethora, petechiae, hirsutism, striae and muscle atrophy.

Published by Bioscientifica Ltd. 